CLINICAL STUDY

\title{
Changes in serum inhibin $B$ during normal male puberty
}

\author{
A F Radicioni, A Anzuini, E De Marco, I Nofroni ${ }^{1}$, V D Castracane ${ }^{2}$ and A Lenzi \\ Clinical Pathology, Department of Medical Pathophysiology and ${ }^{1}$ Medical Statistics and Biometrics, University of Rome, La Sapienza, \\ Italy and ${ }^{2}$ Diagnostic Systems Laboratories, Inc., Webster, Texas, USA \\ (Correspondence should be addressed to A F Radicioni, Laboratory of Endocrinology, Department of Medical Pathophysiology, University of Rome, \\ La Sapienza, Policlinico Umberto I, OO 161 Rome, Italy; Email: antonio.radicioni@uniroma1.it)
}

\begin{abstract}
Objective: In adult men, inhibin B (InhB) regulates FSH secretion by a negative feedback. The aims of this study were to evaluate the changes of $\operatorname{InhB}$ during puberty in the male and the relationship between InhB and FSH, LH, testosterone and testicular volume.

Design: Cross-sectional study.

Methods: InhB was measured using a two-site ELISA in 100 healthy boys subdivided by their pubertal development according to Tanner into five groups of 20.

Results: During puberty we observed an increase of InhB level $(\mathrm{G} 1=84.3 \mathrm{pg} / \mathrm{ml}, \mathrm{G} 3=132.2 \mathrm{pg} / \mathrm{ml}$, $\mathrm{G} 5=206.1 \mathrm{pg} / \mathrm{ml})$. In G1, InhB correlated positively with FSH $(P=0.0001)$, LH $(P=0.005)$, testosterone $(P=0.001)$ and testicular volume $(P=0.007)$; in $\mathrm{G} 5$, InhB correlated inversely with FSH $(P=0.001)$ and LH $(P=0.045)$ and directly with testicular volume $(P=0.013)$. The multivariate analysis demonstrated that: in G1, FSH is the most important, and testosterone the second most significant, stimulus for InhB increase; in G2 only FSH has a positive effect on InhB variation; in G3 only mean testicular volume fits the model (G1-G3: InhB dependent variable); considering the FSH dependent variable, in G4, InhB is the most important stimulus for FSH decrease and mean testicular volume is a secondary directly proportional variable; in G5, only InhB shows a significant inverse relationship with FSH.

Conclusions: During puberty there is a regular increase of InhB. In the first phases of gonadal maturation, InhB and FSH correlate positively, while in mid-late stages the relationship is inverse. We found that in mid-puberty (G3-G4), the serum concentration of InhB increases, as its inverse relationship with FSH is being established and hence spermatogenesis.
\end{abstract}

European Journal of Endocrinology 152 403-409

\section{Introduction}

The onset of male puberty is clinically characterized by an increase in testicular volume, consequent to reactivation of the hypothalamic-pituitary-gonadal (HPG) axis. An adequate follicle-stimulating hormone (FSH) concentration is necessary in the first pubertal stages to induce proliferation and differentiation of Sertoli cells (1). Inhibin B (InhB) is a heterodimeric glycoprotein, consisting of an $\alpha$ - and a $\beta$-subunit covalently linked by a disulfide bond (2). Using an ELISA method specific for the dimeric form, and capable of distinguishing between InhA, InhB and pro- $\alpha \mathrm{C}$-related immunoreactive peptides, an inverse relationship between FSH and InhB was demonstrated in men with both normal and abnormal spermatogenesis (3) and an InhB concentration below the limit of detection in ten patients with therapeutic bilateral orchidectomy (4). The change in InhB value and its relationship with FSH have been studied from birth to puberty. It has been demonstrated that in boys, InhB values peak during the first few months of life and remain at measurable levels during childhood with a nadir at $6-10$ years. This is in contrast with other hormones of the HPG axis: FSH, luteinizing hormone (LH), testosterone and estradiol, which after the neonatal peak decrease to very low levels $(5,6)$. In pre-pubertal boys, human FSH therapy increases InhB production and induces combined Sertoli cell proliferation and elongation of seminiferous tubules (7). At the start of puberty InhB levels increase more rapidly than the other endocrine signals associated with reproduction: a cross-sectional study of 400 healthy boys showed a significant increase in InhB from puberty stage G1 to G2 (8). Crofton et al. (6) demonstrated a significant increase in InhB between phases G2 and G3. By analysis of semen characteristics in pubertal boys, Janczewski \& Bablok $(9,10)$ highlighted the appearance of spermatozoa in the seminal fluid at around 13.5 years and a testicular volume of $10 \mathrm{ml}$ in mid-puberty. The aims of the present study were to evaluate the changes of InhB during male puberty and the relationship between 
InhB and FSH, LH, testosterone and mean testicular volume (MTV), to verify the hypothesis that the increase in InhB concentration during puberty can be correlated with an increase in testicular volume and hence spermatogenesis.

\section{Materials and methods}

\section{Subjects}

The study design included 100 healthy subjects, subdivided by their pubertal development according to Tanner (11) into five groups of 20. The subjects all came under our observation from January to December 2001 for clinical assessment of pubertal development and were at the normal stage of pubertal development for their chronological age. The sign for clinical onset of puberty (G2) was considered as testicular volume $>3 \mathrm{ml}$. Inclusion criteria were the absence of acute and chronic pathologies, in particular uro-andrological pathologies such as cryptorchidism, varicocele and orchiepididymitis, and endocrine pathologies (diabetes and dysthyroidism). All boys had normal free triiodothyronine, free thyroxine, thyrotropin, growth hormone, insulin-like growth factor-I and cortisol concentrations; none showed a bone age more than \pm 1.5 S.D. away from their chronological age. Gonad volume was measured using the Prader orchidometer (12); boys demonstrating greater than $20 \%$ testicular dysmetria were excluded from the study. MTV, FSH, LH, testosterone and InhB were the variables measured. All subjects and their parents were asked for and provided their informed consent.

\section{Hormone assays}

Fasting morning blood samples were taken from patients between 0800 and $0900 \mathrm{~h}$ from an antecubital vein. Samples were centrifuged after $30 \mathrm{~min}$ and the serum immediately frozen at $-80^{\circ} \mathrm{C}$ for $\mathrm{InhB}$ analysis and $-20^{\circ} \mathrm{C}$ for other analyses. Serum InhB was measured in duplicate using a two-site ELISA (Serotec, Oxford, UK). The detection limit was $15.0 \mathrm{pg} / \mathrm{ml}$ and intra- and inter-assay coefficients of variation were 6.3 and $7.9 \%$ respectively at $128 \mathrm{pg} / \mathrm{ml}$ for our laboratory. Serum FSH, LH and testosterone were measured by an immunofluorometric assay (Autodelfia; Perkin-Elmer Life Sciences), with detection limits of $0.05 \mathrm{U} / \mathrm{l}, 0.05 \mathrm{U} / \mathrm{l}$ and $0.3 \mathrm{nmol} / \mathrm{l}$ respectively; intra- and inter-assay coefficients of variation were 3.6 and $5.4 \%$ at $3.2 \mathrm{U} / \mathrm{l}$ (FSH), 3.8 and $5.5 \%$ at $4.1 \mathrm{U} / \mathrm{l}(\mathrm{LH})$ and 2.4 and $4.1 \%$ at $11.5 \mathrm{nmol} / \mathrm{l}$ (testosterone) for our laboratory.

\section{Statistical analysis}

A descriptive statistical analysis was performed on the data obtained to verify their reliability. After having confirmed a Gaussian distribution of the data, an
ANOVA test was applied to compare means of the variables at each pubertal stage. Univariate linear regression was used to examine the relationship between InhB and other variables at each pubertal stage. Following this, a multiple linear regression model was used, with a stepwise selection procedure for the independent variables. On the basis of current knowledge of the InhB action mechanism, InhB was considered as a dependent variable for stages G1-G3, and as an independent variable in G4-G5 on the basis of its documented action on hypophysial FSH secretion. Statistical significance, for all tests used, was at $P<0.05$. Statistical analysis was realized with the SPSS program (SPSS Inc. release. no. 10, 1999).

\section{Results}

In Fig. 1, InhB values are plotted vs age; after 10-11 years there was a regular increase of InhB concentration until $16-17$ years.

\section{Serum InhB, FSH, LH, testosterone and MTV in relation to pubertal stage}

In the scatter diagram (Fig. 2) InhB levels in serum are represented in the 100 subjects divided by pubertal stage. It can be seen that stages G4-G5 show a notable difference in values with respect to the previous stages. ANOVA comparison of the means of the six variables for each pubertal stage demonstrated significant differences for them all. Figure 3 highlights changes in each variable: MTV undergoes a highly significant linear increase throughout pubertal development (in all comparisons $P=0.001)$; testosterone rises very slowly during G1-G2 (not significant) and then jumps rapidly for $\mathrm{G} 2-\mathrm{G} 3 \quad(P=0.001)$ and $\mathrm{G} 3-\mathrm{G} 4 \quad(P=0.001)$; $\mathrm{LH}$, which already seems to reach adult levels by G2, thus anticipating the increase in testicular volume and

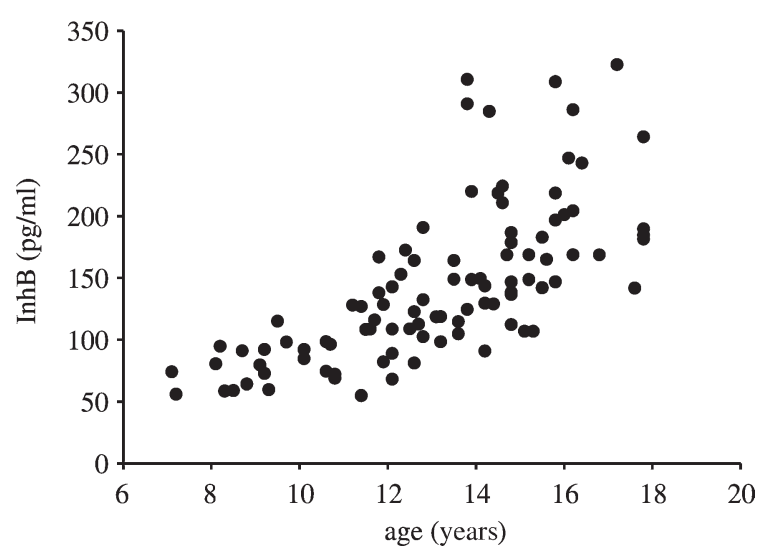

Figure 1 Individual serum concentration of InhB plotted as a function of age in 100 healthy subjects. During childhood the InhB values are relatively constant, after 10-11 years there was a regular increase of InhB concentration until 16-17 years. 


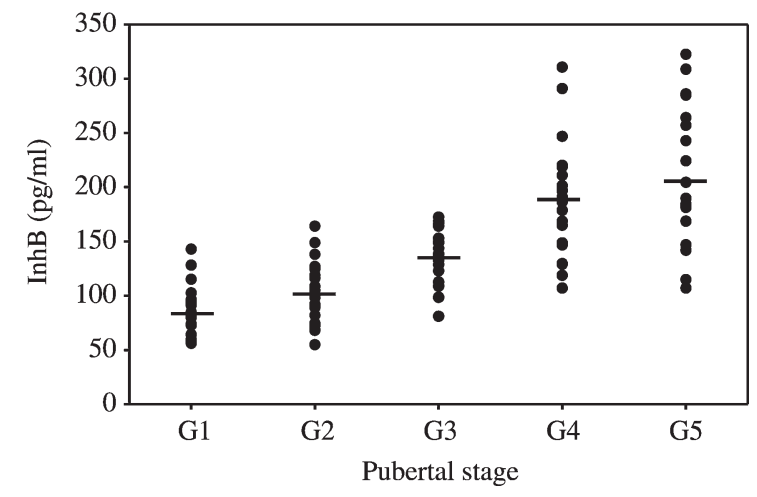

Figure 2 Scatter diagram of InhB values during puberty in 100 healthy subjects, divided by their pubertal stage according to Tanner (11). The mean value of InhB increases particularly from G2 to G3 and from G3 to G4. In the late phases of puberty (G4 and G5), we observed a very large range of concentrations.

testosterone, shows a notable increase from G1 to G2 $(P=0.001)$ and an even more marked increase from G2 to G3 $(P=0.001)$; FSH seems to be the first parameter to notably increase from G1 to G3 (G1 vs G2 $P=0.001$; G2 vs G3 $P=0.016)$, but then remains relatively constant; InhB rises slowly from G1 to G2 (not significant), more rapidly from $\mathrm{G} 2$ to $\mathrm{G} 3(P=0.016)$, and even more rapidly from $\mathrm{G} 3$ to $\mathrm{G} 4(P=0.002)$, with a final, smaller increase from G4 to G5 (not significant). As expected, a marked variability was seen both interand intra-group (Table 1).

\section{Relationship of InhB with age, FSH, LH, testosterone and MTV at different pubertal stages}

The relationships evaluated by simple linear regression (Table 2) highlighted that in G1, InhB has a significant,

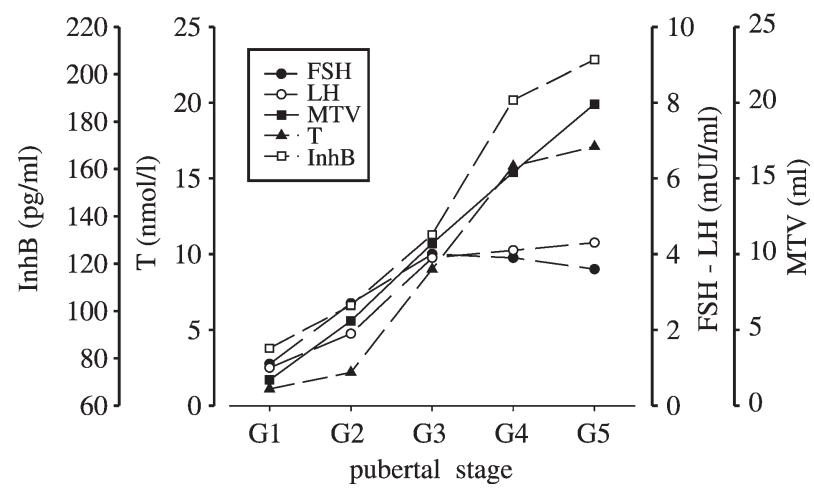

Figure 3 Mean of FSH, LH, testosterone, InhB and MTV, plotted against genital stage in 100 healthy subjects. FSH notably increases from $\mathrm{G} 1$ to $\mathrm{G} 2$ and $\mathrm{G} 2$ to $\mathrm{G} 3$, after remaining at a relatively constant level during late puberty; LH shows a notable increase from $\mathrm{G} 1$ to $\mathrm{G} 2$, and an even more marked increase from $\mathrm{G} 2$ to $\mathrm{G} 3$, after remaining at a relatively constant level during G4 and G5 stages; MTV shows a linear increase during pubertal maturation; testosterone ( $T$ ) rises slowly from $\mathrm{G} 1$ to $\mathrm{G} 2$, increasing rapidly from $\mathrm{G} 2$ to $\mathrm{G} 4$, then rises slowly from $\mathrm{G} 4$ to $\mathrm{G} 5$; InhB rises slowly from $G 1$ to $G 2$, more rapidly from $G 2$ to $G 3$, even more rapidly from $\mathrm{G} 3$ to $\mathrm{G} 4$, and rises slowly from $\mathrm{G} 4$ to $\mathrm{G} 5$. direct dependency on all variables. In G2, InhB has a significant, direct dependency on $\mathrm{FSH}$, testosterone and MTV but not on age and LH. In G3, there was a direct dependency only on MTV. In G4, a highly significant inverse dependency was found with FSH and a modest dependency with testosterone. Finally, in G5, a distinct inverse relationship with FSH remained, but a modest inverse relationship with LH was also seen, as was a direct relationship with the increase in MTV. The complexity of the developmental process and the consequent relationship between diverse hormones suggested the use of the multiple linear regression model. The model previously used was incapable of selecting those variables with a real role in gonadal maturation and of investigating the strong relationship between FSH and InhB documented in other studies. As already noted, InhB was considered as the dependent variable for stages G1-G3, and FSH for G4 and G5 (Table 3, Fig. 4). In G1, FSH is by far the most important variable for determination of positive variations in InhB values $\left(r^{2}=0.54\right)$, while testosterone demonstrated a secondary but significant, directly proportional role (FSH and testosterone: $\left.r^{2}=0.66\right)$. In $\mathrm{G} 2, \mathrm{FSH}$ is the only variable affecting positive variations in InhB $\left(r^{2}=0.46\right)$. In G3, the influence of FSH disappears and the direct influence of MTV is evident $\left(r^{2}=0.16\right)$. Finally, with FSH as the dependent variable, in G4 InhB is the most important variable, with an inverse correlation with FSH concentration $\left(r^{2}=0.50\right)$, while MTV demonstrated a secondary but significant indirectly proportional role (InhB and MTV: $\left.r^{2}=0.61\right)$. In $\mathrm{G} 5$, only InhB has a role, with an inverse relationship with FSH $\left(r^{2}=0.44\right)$.

\section{Discussion}

InhB demonstrates a progressive increase throughout puberty, until it reaches normal adult values as seen in studies of adults with normal spermatogenesis (data not shown). As can clearly be seen in Fig. 2, InhB concentrations show a notable increase in stage G3 and above all in stage G4, in which it stabilizes until the end of puberty (G5). In a cross-sectional study (8) of 400 healthy boys the InhB increase was already documented at stage G2, where it reached levels similar to those seen in adults males and thus did not undergo further alteration during pubertal development. In a recent study Crofton et al. (6) demonstrated a significant and progressive increase in InhB from late pre-puberty (G1) to G3, subsequently observing a small decrease in stages $\mathrm{G} 4$ and G5. In both of these earlier studies, InhB is seen to increase earlier or more dramatically that other endocrine factors. Data from our group seem to shift the progressive InhB increase to a more advanced development stage (G3-G4) than previous studies. Such differences, which may be biologically important, do not seem to be due to the clinical characteristics of the subjects 
Table 1 Descriptive statistical analysis.

\begin{tabular}{|c|c|c|c|c|c|c|}
\hline Pubertal stage & $\begin{array}{c}\text { Age } \\
\text { (years) }\end{array}$ & $\begin{array}{c}\text { MTV } \\
(\mathrm{ml})\end{array}$ & $\begin{array}{l}\text { InhB } \\
(\mathrm{pg} / \mathrm{ml})\end{array}$ & $\begin{array}{c}\text { FSH } \\
(\mathrm{mUl} / \mathrm{ml})\end{array}$ & $\begin{array}{c}\text { LH } \\
(\mathrm{mUl} / \mathrm{ml})\end{array}$ & $\begin{array}{c}\text { Testosterone } \\
(\mathrm{nmol} / \mathrm{l})\end{array}$ \\
\hline \multicolumn{7}{|l|}{ G1 } \\
\hline Mean & 9.3 & 1.7 & 84.3 & 1.1 & 1.0 & 1.1 \\
\hline S.D. & 1.5 & 0.6 & 24.0 & 0.4 & 0.3 & 0.4 \\
\hline Minimum & 7.1 & 1.0 & 55.9 & 0.7 & 0.6 & 0.5 \\
\hline Maximum & 12.8 & 3.0 & 142.8 & 2.2 & 1.9 & 2.2 \\
\hline \multicolumn{7}{|l|}{ G2 } \\
\hline Mean & $11.9^{\mathrm{a}}$ & $5.6^{\mathrm{a}}$ & 102.4 & $2.7^{\mathrm{a}}$ & $1.9^{\mathrm{a}}$ & 2.2 \\
\hline S.D. & 1.2 & 1.2 & 29.0 & 0.7 & 0.4 & 0.6 \\
\hline Minimum & 9.7 & 4.0 & 54.8 & 1.6 & 1.0 & 1.0 \\
\hline Maximum & 14.2 & 7.0 & 164.0 & 4.2 & 2.8 & 3.3 \\
\hline \multicolumn{7}{|l|}{ G3 } \\
\hline Mean & 13.1 & $10.7^{\mathrm{a}}$ & $132.2^{c}$ & $4.0^{c}$ & $3.9^{\mathrm{a}}$ & $9.0^{\mathrm{a}}$ \\
\hline S.D. & 1.2 & 1.6 & 26.4 & 1.4 & 1.1 & 2.1 \\
\hline Minimum & 10.6 & 8.0 & 81.0 & 2.0 & 2.1 & 4.8 \\
\hline Maximum & 14.8 & 14.0 & 172.4 & 7.8 & 5.7 & 12.6 \\
\hline \multicolumn{7}{|l|}{ G4 } \\
\hline Mean & $14.7^{\mathrm{a}}$ & $15.4^{\mathrm{a}}$ & $189.1^{\mathrm{b}}$ & 3.9 & 4.1 & $15.8^{\mathrm{a}}$ \\
\hline S.D. & 0.9 & 2.1 & 53.9 & 1.0 & 1.1 & 6.4 \\
\hline Minimum & 12.8 & 12.0 & 106.8 & 2.1 & 2.0 & 8.9 \\
\hline Maximum & 16.1 & 18.0 & 310.5 & 6.4 & 5.8 & 32.0 \\
\hline \multicolumn{7}{|l|}{ G5 } \\
\hline Mean & $16.3^{\mathrm{a}}$ & $19.9^{\mathrm{a}}$ & 206.1 & 3.6 & 4.3 & 17.1 \\
\hline S.D. & 1.2 & 2.0 & 64.4 & 0.9 & 1.4 & 5.0 \\
\hline Minimum & 13.6 & 15.0 & 106.8 & 2.1 & 2.0 & 8.4 \\
\hline Maximum & 17.8 & 25.0 & 322.4 & 6.2 & 7.0 & 26.4 \\
\hline
\end{tabular}

Statistical significance is indicated when the mean of one puberty stage has a statistically significant difference from the mean of the previous stage (by ANOVA test).

${ }^{\mathrm{a}} P=0.001 ;{ }^{\mathrm{b}} P=0.002 ;{ }^{\mathrm{c}} P=0.016$.

Table 2 Simple linear regression between $\operatorname{lnhB}$ (dependent variable) and the other variables at each stage.

\begin{tabular}{|c|c|c|c|c|}
\hline Pubertal stage & Independent variables & Adjusted $r$ square & Beta & $t$-test $P$ value \\
\hline \multirow[t]{5}{*}{ G1 } & $\mathrm{FSH}$ & 0.54 & 41.74 & 0.0001 \\
\hline & $\mathrm{LH}$ & 0.33 & 43.62 & 0.005 \\
\hline & Testosterone & 0.44 & 0.06 & 0.001 \\
\hline & MTV & 0.30 & 23.64 & 0.007 \\
\hline & Age & 0.48 & 11.61 & 0.0001 \\
\hline \multirow[t]{5}{*}{ G2 } & FS̈H & 0.46 & 27.96 & 0.001 \\
\hline & $\mathrm{LH}$ & 0.02 & 20.24 & n.s. \\
\hline & Testosterone & 0.16 & 0.82 & 0.044 \\
\hline & MTV & 0.39 & 15.80 & 0.002 \\
\hline & Age & 0.13 & 9.75 & n.s. \\
\hline \multirow[t]{5}{*}{ G3 } & FS̆H & -0.05 & 1.45 & n.s. \\
\hline & $\mathrm{LH}$ & -0.01 & 5.49 & n.s. \\
\hline & Testosterone & 0.07 & 0.15 & n.s. \\
\hline & MTV & 0.16 & 7.30 & 0.047 \\
\hline & Age & 0.04 & 6.47 & n.s. \\
\hline \multirow[t]{5}{*}{ G4 } & $\mathrm{FSH}$ & 0.50 & -38.13 & 0.0001 \\
\hline & $\mathrm{LH}$ & -0.03 & -8.15 & n.s. \\
\hline & Testosterone & 0.18 & 0.14 & 0.034 \\
\hline & MTV & -0.04 & 3.15 & n.s. \\
\hline & Age & -0.06 & -1.66 & n.s. \\
\hline \multirow[t]{5}{*}{ G5 } & FS̆H & 0.44 & -46.78 & 0.001 \\
\hline & LH & 0.16 & -20.86 & 0.045 \\
\hline & Testosterone & 0.01 & 0.12 & n.s. \\
\hline & MTV & 0.26 & 17.39 & 0.013 \\
\hline & Age & -0.03 & 7.98 & n.s. \\
\hline
\end{tabular}


Table 3 Multiple linear regression at each pubertal stage.

\begin{tabular}{llclcc}
\hline Pubertal stage & Dependent variable & Adjusted $\boldsymbol{r}$ square & Variables on the model & Beta & $\boldsymbol{t}$-test $\boldsymbol{P}$ value \\
\hline G1 & InhB & 0.54 & FSH & 30.42 & 0.002 \\
G2 & & 0.66 & Testosterone & 0.81 & 0.015 \\
G3 & InhB & 0.46 & FSH & 27.96 & 0.001 \\
G4 & InhB & 0.16 & MTV & 7.3 & 0.047 \\
& FSH & 0.50 & InhB & -0.013 & 0.0001 \\
G5 & FSH & 0.61 & MTV & -0.17 & 0.028 \\
& & 0.44 & InhB & -0.01 & 0.001 \\
\hline
\end{tabular}

Pubertal stage G1

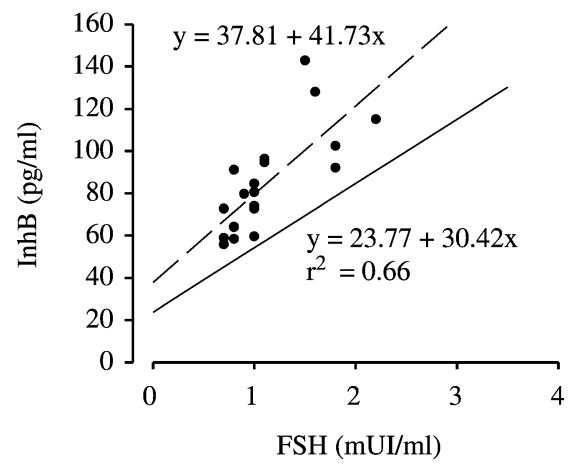

Pubertal stage G4

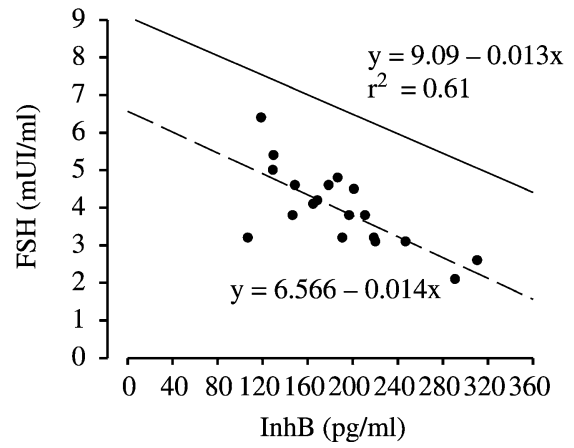

Pubertal stage G2

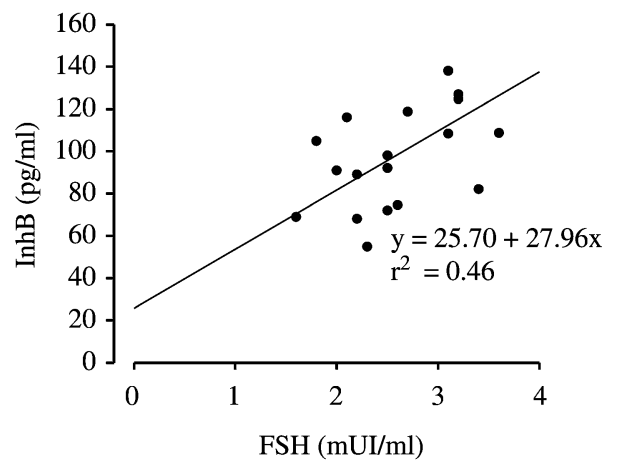

Pubertal stage G5

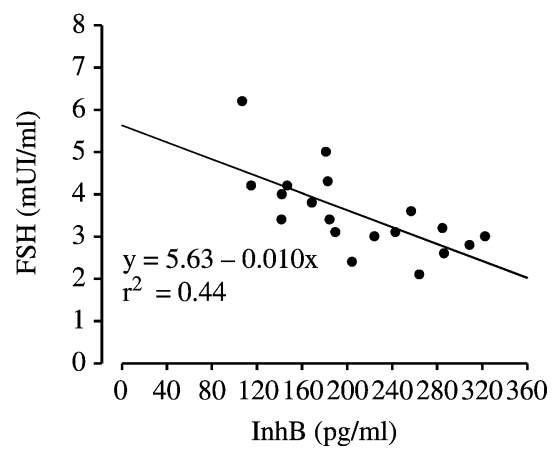

Figure 4 Multiple linear regression at different pubertal stages. Pubertal stage G1: regression line between InhB (dependent variable) and $\mathrm{FSH}$, the first variable selected from the multivariate model (broken line); the solid line indicates the straight line equation when the model also considers the second explicative variable (testosterone). Pubertal stage G2: regression line between InhB (dependent variable) and $\mathrm{FSH}$, the only independent variable selected from the multivariate model. Pubertal stage G4: regression line between FSH (dependent variable) and InhB, the first variable selected from the multivariate model (broken line); the solid line indicates the straight line equation when the model also considers the second explicative variable (testosterone). Pubertal stage G5: regression line between FSH (dependent variable) and InhB, the only independent variable selected from the multivariate model. In this Figure, the pubertal stage G3 line has not been represented because only MTV fits the model, with a small contribution to the prediction of InhB values. In the first stages (G1 and G2) both the variables positively correlate; in G4 and G5 variables negatively correlate.

placed into the various pubertal stages. It can also be seen that in the present study the number in the various groups is the same, whereas in the two previously cited studies there was a clear difference, with a strong stage G1 and G5 prevalence in Andersson's study (8) and prevalence of G1 alone in Crofton's work (6). Furthermore, the difference between our study and previous works may relate to timing of the blood samples, because at $0800-0900 \mathrm{~h}$ FSH values relate more closely to overnight pulsatile secretion. This may have uncovered a relationship with InhB that was not picked up by earlier studies. In the middle phase of male pubertal maturation (G3) we observed an acceleration in the growth of testicular volume preceding the spurt in height. This is the most delicate and important moment, as meiosis is completed and gametogenesis proceeds until more mature stages are reached (spermarche). This is in general agreement with Nielsen 
et al. (13), who reported that spermarche occurred at a median age of 13.4 years (range $11.7-15.3$ years) with a median estimated testicular volume of $11.5 \mathrm{ml}$ (wide range from 4.7 to $19.6 \mathrm{ml}$ ), and a median pubic hair stage of 2.5 (range stages $1-5$ ).

Our data show a strong positive relationship between FSH (as the independent variable) and InhB in pre-puberty and the initial stages of gonadal maturation. Previously reported data (8) show a more irregular progress of the correlation between FSH and InhB, with a positive statistical correlation only at stage IB, consisting of boys aged 9 or above, and not at stages IA or II. However, when the effect of age was considered at stage IB, the statistical significance disappeared. The difference between our results and previous studies, at least for stage G1, may be due to the age of our subjects, who were referred for evaluation of pubertal development and especially of the level of hypophysis activation, on average more advanced (higher mean FSH and MTV) than in those studied by the Danish group (8). In any case, taken together the results demonstrate that the positive relationship may begin on the initiation of FSH gonad stimulation, immediately before any clinical evidence of the start of puberty. From this point of view the direct action of FSH on the Sertoli cells determines the increase in InhB secretion highlighted in all the studies and could thus explain the positive correlation we also found in stage G2 between FSH and InhB. Our analysis also suggests that FSH may be the most important stimulus for InhB production, in agreement with the data of Andersson et al. (14). During pubertal maturation the relationship inverts and becomes negative, as seen in adult males. Our results, in agreement with previous data (6), indicate that this occurs in mid-puberty, phase G3. We have hypothesized that this stage contains boys with their spermatogenesis in evolution, but partially differing, and that it should therefore be possible to find both subjects with a still positive correlation, and those in whom it has already inverted. The global evaluation of these two types, with functionally diverse gonadal maturation although both clinically and auxologically categorized as stage G3, leads to the absence of correlation seen here. In fact, the multiple linear regression model demonstrated a significant positive correlation only with the MTV. It may be possible to subdivide phase G3 into early-G3 and late-G3 on the basis of testicular volume $(\mathrm{G} 3 \mathrm{~A}$ : MTV $\leq 10 \mathrm{ml}$; G3B: MTV $>10 \mathrm{ml}$ ), which would indicate when this developmental change has taken place.

The relationship found between InhB and MTV at G5 may be related to spermatogenesis levels for the demonstrated relationship between InhB and sperm concentration in men with normal spermatogenesis (15).

It is interesting to note that the negative effect of MTV on FSH values highlighted by the multivariate analysis model, unmediated by InhB, the main variable, could be tied to testicular follistatin production (16).
The early increase in FSH concentration stimulates the Sertoli cells and induces the increase in serum InhB levels, in late pre-puberty (stage G1) and in early puberty (G2): this determines the pubertal phase of Sertoli cells proliferation and differentiation (1). During this period, as in the neonatal phase, there is a strong positive correlation between FSH and InhB.

In contrast, InhB increase in mid-puberty (G3-G4) coincides with the maturation of the male gonad and completion of spermatogenesis, and is characterized in normal individuals by the appearance of a significant negative correlation between InhB and FSH. This seems in agreement with Andersson's data (13), which demonstrated for pre-pubertal testes an intense, positive immunostaining for inhibin $\alpha$-subunit and $\beta B$-subunit in Sertoli cells, while in adult testes, immunostaining for inhibin $\alpha$-subunit only was observed in Sertoli and Leydig cells, with no staining observed for the inhibin $\beta \mathrm{B}$-subunit, located in germ cells from pachytene spermatocytes to round spermatids.

In summary, our study has confirmed a strong biphasic relationship of InhB with FSH during pubertal development, with the inversion of this relationship occurring in mid-puberty with the start of spermatogenesis. InhB levels increase regularly during puberty until they reach adult levels in late puberty (G4-G5). This increase mirrors the progressive increase in testicular volume and spermatogenesis occurring in late puberty.

On the basis of literature data and these results, we can hypothesize that during late pre-puberty and early puberty, InhB is produced by Sertoli cells and may have an essentially autocrine and paracrine role in the increase and differentiation of Sertoli and Leydig cells and spermatogonia, whereas during successive pubertal stages InhB is produced by spermatogenesis cells with the Sertoli cells and its action is essentially endocrinal, as a negative feedback on the FSH-secreting pituitary cells. Further studies are in progress on models of human gonadal pathologies, to understand the mechanisms through which these changes in InhB action occur.

\section{Acknowledgements}

We acknowledge the general advice of Scott Bishop (Diagnostic Systems Laboratories, Inc.)

\section{References}

1 Cortes D, Müller J \& Skakkebœk NE. Proliferation of Sertoli cells during development of the human testis assessed by stereological methods. International Journal of Andrology $1987 \mathbf{1 0} 589-596$.

2 De Kretser DM \& McFarlane JR. Inhibin in the male. Journal of Andrology 199617 179-182.

3 Illingworth PJ, Groome NP, Bryd W, Rainey WE, McNeilly AS, Mather JP \& Bremner WJ. Inhibin-B: a likely candidate for the 
physiologically important form of inhibin in men. Journal of Clinical Endocrinology and Metabolism 199681 1321-1325.

4 Anawalt BD, Bebb RA, Matsumato AM, Groome NP, Illingworth PJ, McNeilly AS \& Bremner WJ. Serum inhibin B levels reflect Sertoli cell function in normal men and men with testicular dysfunction. Journal of Clinical Endocrinology and Metabolism $1996813341-3345$.

5 Andersson AM, Toppari J, Haavisto AM, Petersen JH, Simell T, Simell O \& Skakkebœk NE. Longitudinal reproductive hormone profiles in infants: peak of inhibin B levels in infant boys exceeds levels in adult men. Journal of Clinical Endocrinology and Metabolism $1998 \mathbf{8 3}$ 675-681.

6 Crofton PM, Evans AEM, Groome NP, Taylor MRH, Holland CV \& Kelnar CJH. Inhibin B in boys from birth to adulthood: relationship with age, pubertal stage, FSH and testosterone. Clinical Endocrinology $200256215-221$.

7 Raivio T, Toppari J, Perheentupa A, McNeilly AS \& Dunkel L. Treatment of pubertal gonadotrophin-deficient boys with recombinant human follicle-stimulating hormone. Lancet $1997 \mathbf{3 5 0}$ 263-264.

8 Andersson AM, Anders J, Petersen JH, Müller J, Groome NP \& Skakkebœek NE. Serum inhibin B in healthy pubertal and adolescent boys: relation to age, stage of puberty and follicle-stimulating hormone, luteinizing hormone, testosterone, and estradiol levels. Journal of Clinical Endocrinology and Metabolism $1997 \mathbf{8 2}$ 3976-3981.

9 Janczewski Z \& Bablok L. Semen characteristics in pubertal boys. II. Semen quality in relation to bone age. Archives of Andrology $198515207-211$.
10 Janczewski Z \& Bablok L. Semen characteristics in pubertal boys. III. Semen quality and somatosexual development. Archives of Andrology 198515 213-218.

11 Tanner JM. Growth at Adolescence. Oxford: Blackwell Science 1962.

12 Zachmann M, Prader A, Kind HP \& Haflinger H. Testicular volume during adolescence. Cross-sectional and longitudinal studies. Helvetica Paediatrica Acta 197429 61-72.

13 Nielsen CT, Skakkebœk NE, Richardson DW, Darling JAB, Hunter WM, Jørgensen M, Nielsen A, Ingerslev O, Keiding N \& Müller J. Onset of the release of spermatozoa (spermarche) in boys in relation to age, testicular growth, pubic hair, and height. Journal of Clinical Endocrinology and Metabolism $1986 \mathbf{6 2} 532$ - 535.

14 Andersson AM, Müller J \& Skakkebœk NE. Different roles of prepubertal and postpubertal germ cells and Sertoli cells in the regulation of serum inhibin B levels. Journal of Clinical Endocrinology and Metabolism $1998 \mathbf{8 3} 4451-4458$.

15 Klingmüller D \& Haidl G. Inhibin B in men with normal and disturbed spermatogenesis. Human Reproduction 199712 2376-2378.

16 Foster CM, Olton PR, Racine MS, Phillips DJ \& Padmanabhan V. Sex differences in FSH-regulatory peptides in pubertal age boys and girls and effects of steroid treatment. Human Reproduction $2004191668-1676$.

Received 23 July 2004

Revised 22 November 2004 